\title{
Peritonitis secondary to a blocked perforated ulcer: management
}

\author{
Amine Chaabouni ${ }^{1}$, Haroun Guermazi ${ }^{1}$, Mohamed Ali Mseddi ${ }^{1}$, and kais fourati ${ }^{2}$ \\ ${ }^{1}$ Habib Bourguiba Hospital \\ ${ }^{2}$ University of Sfax Faculty of Medecine of Sfax
}

January 10, 2022

\begin{abstract}
We report the case of a 42 years old young patient who was examined in the emergency department for epigastric pain lasting for 3 days. The abdominal tomodensitometry allowed to highlight the presence of an intra peritoneal effusion without pneumopertoneum. The patient underwent an exploratory laparoscopy. There was peritoneal toilet
\end{abstract}

\section{Peritonitis secondary to a blocked perforated ulcer: management}

Amine Chaabouni1,a. Haroun Guermazi1. Mohamed Ali Mseddi1. Kais Fourati1

General Surgery Department, Habib Bourguiba Hospital, University of Sfax, 3029 Sfax, Tunisia

a Corresponding author: amine_chaabouni@yahoo.com

Written informed consent was obtained from the patient to publish this report.

Introduction:

Peptic ulcer disease (PUD) is a common request for medical care. Most often benign, they are sometimes complicated by hemorrhage or perforation, which can drastically alter the prognosis. In this report, we will concern ourselves with sealed perforated ulcers. In the context of actual trends towards an efficient and non aggressive treatment as surgery carries additional morbid-mortality, this condition generates a therapeutic debate between those who favor a preventive strategy aimed at operating the patient in order to avoid the death; and those who follow a conservative strategy which only refers the patient to the operating theatre in the case of deterioration. We will be reporting on our own experience with a contained perforated duodenal ulcer which was operated on secondarily after non-response to medical treatment.

Case report:

Patient N D, 42 years old, smoker with no pathological history, presented to his primary care physician complaining of cramp epigastralgia evolving during 03 days. The biology showed leukocytes $=15.3^{*} 10^{\wedge} 3 / \mathrm{mm} 3$ of which $65 \%$ PNN, hemoglobin= $17.5 \mathrm{~g} / 100 \mathrm{~mL}$ platelets $=343^{*} 10^{\wedge} 3 / \mathrm{mm} 3$, C-reactive protein $(\mathrm{CRP})=2 \mathrm{mg} / \mathrm{L}$ $\mathrm{PCT}<0.05 \mathrm{ng} / \mathrm{mL}, \mathrm{D}$-dimer $=1755.710 \mathrm{ng} / \mathrm{mL}$ troponin $=1,5 \mathrm{ng} / \mathrm{L}$, lipase $=33 \mathrm{UI} / \mathrm{L}$, urea $=2 \mathrm{mmol} / \mathrm{L}$, Prothrombin Ratio $=98 \%$, plain abdominal $X$-ray $=$ no pneumoperitoneum. He had a symptomatic medical treatment. The evolution was marked by the persistence of the pain, dyspnea and sinus tachycardia at 140ppm. He was tested for SARS Covid by PCR with a nasopharyngeal swab which came back negative. An abdominal thoraco CT scan without injection showed a pulmonary parenchymal condensation in the apical segment of the right lower lobe, atelectasis band under the basal segment bilaterally, spasmodic aspect of the antropyloric region with satellite adenomegaly with small size, diverticula of the sigmoid and the right colon. A transthoracic echocardiography showed a non-dilated non hypertrophied left ventricle (LV) with moderate decrease of the systolic function of the $\mathrm{LV}(\mathrm{LVEF}=58 \%)$, pericarditis of low abundance. He was put under 
bisoprolol then ivabradine for a better control of the cardiac frequency. Considering the non decrease of his abdominal pain after $48 \mathrm{~h}$ and the association of fever and vomiting, he had an upper digestive fibroscopy which revealed a very inflammatory aspect of the stomach with big soft and oedematized antrofindic folds, permeable pylorus, very inflammatory bulb with presence of a big bulbic ulcer quite excavated occupying the small curvature and the anterior face of the bulb with inflammatory narrowing of the bulbar top which remains crossed with hard friction by the fiberscope. A complement of injected abdominal CT scan and with high digestive opacification with water-soluble showed the appearance of disorders of ventilation of the two bases associated with a right pleural effusion, the appearance of a peri-hepatic effusion and at the level of the right parieto colic gutter with densification of the mesenteric fat next to the gastric antrum without leak of contrast agent in extra digestive (figure 1). He was put under nasogastric aspiration, infusion, cefotaxime, metronidazole, gentamicin, double dose of omeprazole and surveillance in an intensive care unit. After 24 hours, in front of the persistence of febrile state and guarding in the physical exam, we decided to operate by laparoscopic approach: the intraoperative exploration showed a diffuse purulent intraperitoneal effusion with multiple false membranes barring the duodenal bulb, abscess under hepatic - under right diaphragmatic and in Douglas' fornix. Appendix and gall bladder were apparently healthy. He had an aspiration of the purulent contents and the flattening of the abscessed collections. Methylene blue test turned out to be negative. The abundant toilet with physiological serum was accompanied by installation of drainage in right sub phrenic, under left phrenic, under hepatic and in the pouch of Douglas. The evolution was marked by apyrexia, restoration of transit on day 01 postoperative; the left sub phrenic drain brought back regularly $100 \mathrm{~mL}$ sero-hematic for 04 days. Other drains only brought back $100 \mathrm{~mL}$ sero-hematic on the first day, drop in leukocytes to 11.000 and CRP to 145.7. Methylene blue test was made on day 04 postoperative, in front of the negative result, the gastric tube was removed and he was allowed to eat. The drainage was removed the next day considering food tolerance. He returned to his home on Day 06 postoperative. He had an eradication of helicobacter pylori. He was seen 02 weeks after, no functional complaints were reported.

Discussion:

The ulcer generates pain in the form of cramps in the pit of the stomach, more rarely burning sensations. They are generally triggered by food intake. Non-steroidal anti-inflammatory drugs (NSAIDs) and the Helicobacter pylori bacteria alone are responsible for the vast majority of ulcers. In fact, a quarter of chronic NSAID users will develop PUD [1]. Although the incidence of PUD has decreased in recent years due to the eradication treatment for Helicobacter pylori and the use of proton pump inhibitors [2], complications of PUD such as perforation and bleeding have remained fairly constant[3]. This may be related to increased use of NSAIDs and to the aging population [4].

The anterior surface of the 1st duodenum is the most prevalent location $(60 \%)$ before the gastric antrum (20\%) and then the lesser gastric curve (20\%)[5].

Duodenal perforations can either be free or contained. Free perforation arises when bowel contents leak freely into the abdominal cavity and causing diffuse peritonitis. Contained perforation occurs when the ulcer creates a full-thickness hole, but free leakage is prevented by contiguous organs such as the pancreas wall off the area[6]. Perforated ulcers may seal spontaneously with fibrin, omentum or by fusion of the duodenum to the underside of the liver between the gallbladder and the falciform ligament.

Considering the phagocytic capacities of the peritoneum and the frequent sterility of the effused fluid, and the fact that the perforation "covered" itself most of the time, allowed surgical treatment to be omitted.

This led E. \Y. Bedforil-Turner in 1945 to propose a conservative treatment consisting of morphine to ease the pain, emptying of the stomach, and permanent aspiration. These measures were then consolidated by H. Taylor the following year, after the experience of 28 cases, which also made it possible to specify the indications[7].

The technique consists of: administration of intravenous analgesic treatment, aspiration of tummy contents, hydration of the patient by parenteral or rectal route, by introducing the quantity of liquid withdrawn from the stomach plus 1 and $1 / 2$ to 2 liters. 
Taylor's results are sensational: among the 28 cases enrolled in the first Taylorization study, 24 uneventful recoveries, 3 deaths for non-digestive reasons, and only one peritonitis by reopening of the sealed ulcer in an undisciplined subject who had drunk after the perforation. It should be added that two cases had to be operated on secondarily when confronted with the finding of contracture persisting.

These results are corroborated by recent results emanating from the prospective study of Cristina Dascalescu and al. [8]which evaluated the security of Taylor's treatment. Among 64 patients, $89 \%$ responded to medical treatment with no need to invasive action. Among 4 patients who developed intra-abdominal abscesses only 2 required drainage but no additional mortality was noted.

With a success rate of around $85 \%$, a further study led by Ruangsak Nusree published in The THAI Journal of SURGERY. He has highlighted that surgery can be prevented.

In the light of the reassuring results of these studies and the fact that the perforation could close spontaneously by filling it by the neighboring organs (liver, gallbladder, round ligament, epiploon), which occurs in about $50 \%$ of cases [9], medical treatment can be safely pursued.

These authors do not propose to systematically renounce surgical treatment of perforated ulcers, but they grant the conservative method the following indications

- Patient seen shortly after perforation (maximum 6 hours);

- Perforation occurring long after a meal, when the stomach can be assumed to be empty;

- General condition too precarious to undergo the operation;

- Distance from a surgical centre (ships at sea, etc.).

This method recognizes the opposite contraindications:

- Late patients;

- Patients who have been drinking after the perforation;

- Suspicion of an abundant peritoneal effusion;

- Gastric stasis due to pyloric stenosis;

- Uncertain location of the ulcer;

- Repeated perforation: operate on the second, without waiting for a third one!

Non-operative treatment may fail and lead to the constitution of a suppuration neighboring the perforation or spreading into the large peritoneal cavity. With the spillage of intraluminal contents into the peritoneal cavity, chemical peritonitis occurs. This is followed by a systemic inflammatory response syndrome (SIRS), which can progress to secondary bacterial peritonitis and sepsis [10].

The treatment thus proposed will join the usual attitude in front of an ulcerous peritonitis: a cleansing associated with an exclusion of the perforation.

In our case, no gesture was performed on the origin of the peritonitis, only a meticulous cleansing was proposed. Our judgment was based on the principle of the physiological defense mechanisms as described above and the worry of inflicting further contamination if an attempt was made to expose the perforation if the abscess cavity bursts during surgery.

Eradication of H. pylori was prescribed considering the fact that H.pylori prevalence ranges are from $50 \%$ to $80 \%$ in patients with perforated duodenal ulcers [11]. In addition, $85.3 \%$ percent of ulcers were healed in the triple therapy group as opposed to $48.4 \%$ in the omeprazole alone group as mentioned by Kin Tong Chung and Vishalkumar G Shelat [1]. 
We did not perform an endoscopy follow-up as it is not recommended due to the low risk of malignancy in such patients [12].

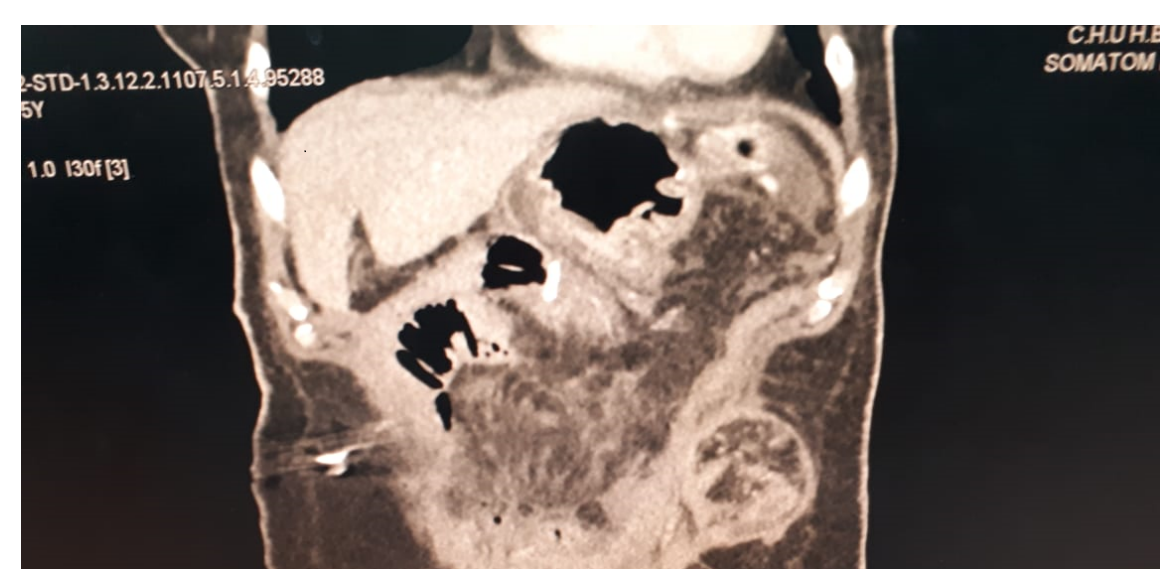

Figure1: Abdominal CT scan( with contrast medium injection) showing peritonitis.

References:

[1] K.T. Chung, V.G. Shelat, Perforated peptic ulcer - an update, WJGS. 9 (2017) 1. https://doi.org/10.4240/wjgs.v9.i1.1.

[2] P. Malfertheiner, F. Megraud, C. O’Morain, F. Bazzoli, E. El-Omar, D. Graham, R. Hunt, T. Rokkas, N. Vakil, E.J. Kuipers, Current concepts in the management of Helicobacter pylori infection: the Maastricht III Consensus Report, Gut. 56 (2007) 772-781. https://doi.org/10.1136/gut.2006.101634.

[3] B. Kocer, S. Surmeli, C. Solak, B. Unal, B. Bozkurt, O. Yildirim, M. Dolapci, O. Cengiz, Factors affecting mortality and morbidity in patients with peptic ulcer perforation, J Gastroenterol Hepatol. 22 (2007) 565570. https://doi.org/10.1111/j.1440-1746.2006.04500.x.

[4] K. Søreide, K. Thorsen, J.A. Søreide, Strategies to improve the outcome of emergency surgery for perforated peptic ulcer, Br J Surg. 101 (2014) e51-64. https://doi.org/10.1002/bjs.9368.

[5] M. Imhof, S. Epstein, C. Ohmann, H.-D. Röher, Duration of Survival after Peptic Ulcer Perforation, World J Surg. 32 (2008) 408-412. https://doi.org/10.1007/s00268-007-9370-2.

[6] A. Amini, R. Lopez, Duodenal Perforation, StatPearls. (2021). https://www.statpearls.com/ArticleLibrary/viewarticle/20757 (accessed October 20, 2021).

[7] H. Taylor, Perforated peptic ulcer; treated without operation, Lancet. 2 (1946) 441-444. https://doi.org/10.1016/s0140-6736(46)90971-3.

[8] C. Dascalescu, L. Andriescu, C. Bulat, R. Danila, L. Dodu, M. Acornicesei, C. Radulescu, Taylor's method: a therapeutic alternative for perforated gastroduodenal ulcer, Hepatogastroenterology. 53 (2006) 543-546.

[9] A.J. Donovan, T.V. Berne, J.A. Donovan, Perforated duodenal ulcer: an alternative therapeutic plan, Arch Surg. 133 (1998) 1166-1171. https://doi.org/10.1001/archsurg.133.11.1166.

[10] D. Ansari, W. Torén, S. Lindberg, H.-S. Pyrhönen, R. Andersson, Diagnosis and management of duodenal perforations: a narrative review, Scandinavian Journal of Gastroenterology. 54 (2019) 939-944. https://doi.org/10.1080/00365521.2019.1647456.

[11] J.P. Gisbert, J.M. Pajares, Helicobacter pylori infection and perforated peptic ulcer prevalence of the infection and role of antimicrobial treatment, Helicobacter. 8 (2003) 159-167. https://doi.org/10.1046/j.15235378.2003.00139.x. 
[12] K. Søreide, K. Thorsen, E.M. Harrison, J. Bingener, M.H. Møller, M. Ohene-Yeboah, J.A. Søreide, Perforated peptic ulcer, Lancet. 386 (2015) 1288-1298. https://doi.org/10.1016/S0140-6736(15)00276-7.

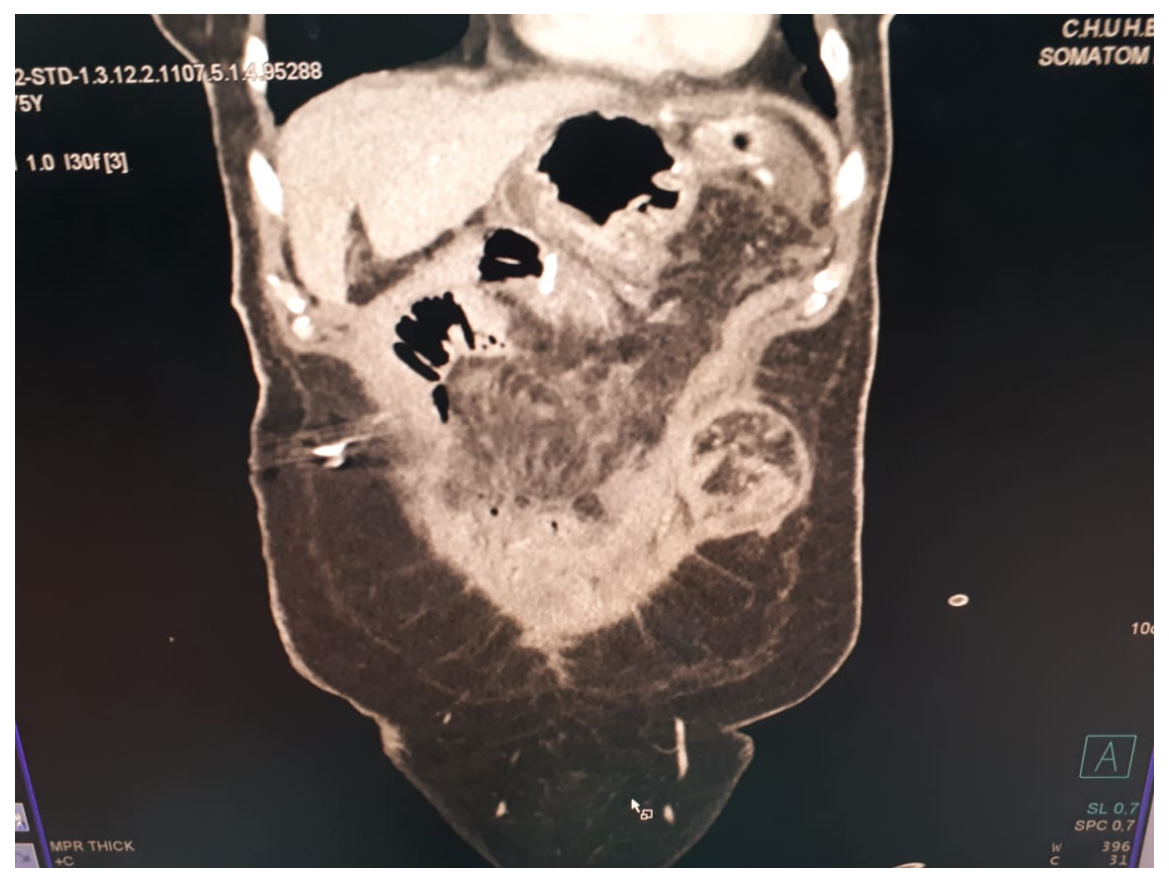

